\title{
Heteroepitaxial integration of InAs/InAsSb type-II superlattice barrier photodetectors onto silicon
}

Peter J. Carrington ${ }^{\mathrm{a}}$, Evangelia Delli ${ }^{\mathrm{a}}$, Veronica Letka ${ }^{\mathrm{b}}$, Matt Bentley ${ }^{\mathrm{a}}$, Peter D. Hodgson ${ }^{\mathrm{b}}$, Eva Repiso $^{\mathrm{b}}$, Jonathan P. Hayton ${ }^{\mathrm{a}}$, Adam P. Craig ${ }^{\mathrm{b}}$, Qi Lu ${ }^{\mathrm{b}}$, Richard Beanland ${ }^{\mathrm{c}}$, Anthony Krier ${ }^{\mathrm{b}}$ and Andrew R. J. Marshall ${ }^{\mathrm{b}}$

(a) Engineering Department, Lancaster University, Bailrigg, Lancaster, LA1 4YW, UK

(b) Physics Department, Lancaster University, Bailrigg, Lancaster, LA1 4YB, UK

(c) Physics Department, University of Warwick, Coventry, CA4 7AL, UK

\begin{abstract}
GaSb-based materials can be used to produce high performance photonic devices operating in the technologically important mid-infrared spectral range. Direct epitaxial growth of $\mathrm{GaSb}$ on silicon $(\mathrm{Si})$ is an attractive method to reduce manufacturing costs and opens the possibility of new applications, such as lab-on-a-chip MIR photonic integrated circuits and monolithic integration of focal plane arrays (FPAs) with Si readout integrated circuits (ROICs). However, fundamental material dissimilarities, such as the large lattice mismatch, polar-nonpolar character of the III-V/Si interface and differences in thermal expansion coefficients lead to the formation of threading dislocations and antiphase domains, which effect the device performance. This work reports on the molecular beam epitaxial growth of high quality GaSb-based materials and devices onto Si. This was achieved using a novel growth procedure consisting of an efficient AlSb interfacial misfit array, a two-step GaSb growth temperature procedure and a series of dislocation filter superlattices, resulting in a low defect density, anti-phase domain free GaSb buffer layer on $\mathrm{Si}$. A nBn barrier photodetector based on a type-II InAs/InAsSb superlattice was grown on top of the buffer layer. The device exhibited an extended $50 \%$ cut-off wavelength at $5.40 \mu \mathrm{m}$ at $200 \mathrm{~K}$ which moved to $5.9 \mu \mathrm{m}$ at $300 \mathrm{~K}$. A specific detectivity of $1.5 \times 10^{10}$ Jones was measured, corresponding in an external quantum efficiency of $25.6 \%$ at $200 \mathrm{~K}$.
\end{abstract}

Keywords: Mid-infrared, photodetector, InAsSb, Molecular beam epitaxy, Photoluminescence, Silicon Photonics

\section{INTRODUCTION}

Type II InAs/InAsSb superlattices (SLs) have emerged as a promising material system for the absorber layer of high efficiency detectors. The type-II band alignment provides remarkable band structure flexibility and the ability to tune the bandgap across the entire infrared wavelength range $^{1}$, while suppressing non-radiative Auger recombination ${ }^{2}$. Conventional InAs/GaSb type-II SLs suffer with reduced minority carrier lifetimes due to the presence of $\mathrm{Ga}$, which is associated with the formation of native defects ${ }^{3,4}$. The use of a Ga-free $\mathrm{nBn}$ architecture using type-II SLs overcomes this problem, whilst also providing further flexibility and functionality. A wide-bandgap barrier layer is placed between the narrow bandgap absorption and contact layers which blocks the flow of majority carriers (electrons), but not that of minority carriers (holes). This almost entirely eliminates the electric field in the narrow-gap material, which greatly suppresses the junction related Shockley-Read-Hall (SRH) dark current, meaning that the $\mathrm{nBn}$ design is less sensitive to crystal defects and, therefore, is well-suited for deposition on a lattice mismatched substrate. Subsequently we demonstrated a high performance InAs/InAsSb SL nBn photodetector on $\mathrm{Si}^{5}$. In this work, we provide a detailed study on the optical and electrical properties to determine the dominant recombination parameters. 


\section{EXPERIMENTAL PROCEDURES}

Samples were grown using a solid source Veeco GENxplor MBE system equipped with valved arsenic (As) and antimony (Sb) cracker cells. N-type Si substrates were used with a $4^{\circ}$ miscut towards the [0-11] direction. An in-situ thermal, chemical free cleaning technique was used prior to epitaxial growth. The thermal treatment consisted of several thermal cycles with the maximum temperature reaching $1000{ }^{\circ} \mathrm{C}$. Such high temperature results in complete desorption of the native oxide and diffusion of carbon contaminants into the bulk of the substrate, leaving a flat Si surface as a result of the high mobility of the $\mathrm{Si}$ atoms. The wafer is then cooled down to the growth temperature of $490{ }^{\circ} \mathrm{C}$ using a fast cooling rate of $\sim 1{ }^{\circ} \mathrm{C} / \mathrm{sec}$ to suppress the thermal segregation of carbon contaminants back to the surface. The surface is then exposed to an $\mathrm{Sb}$ flux for 5 minutes followed by the deposition of a thin 17 monolayer (ML) AlSb nucleation layer, using a growth rate of $0.36 \mathrm{ML} / \mathrm{s}$. Exact control of the AlSb layer creates an efficient interfacial misfit (IMF) array, preventing coalescence of the AlSb islands, which can lead to the possible creation of planar defects such as antiphase domains (APDs) and twins ${ }^{6}$. A $2 \mu \mathrm{m}$ thick GaSb buffer layer was then grown using a two-step growth temperature technique with a Ga growth rate of $0.66 \mathrm{ML} / \mathrm{sec}$. This two-step structure consisted of a $1.5 \mu \mathrm{m}$ thick GaSb layer grown at the same temperature as the AlSb layer $\left(490{ }^{\circ} \mathrm{C}\right)$ in order to maintain the shape of the AlSb islands and ensure planar $2 \mathrm{D}$ growth. Then, the temperature was increased to $515^{\circ} \mathrm{C}$, which in this work is considered the optimum growth temperature for high crystalline quality $\mathrm{GaSb}$ on $\mathrm{Si}$, and a further $500 \mathrm{~nm}$ of $\mathrm{GaSb}$ was deposited. The $\mathrm{GaSb}$ growth temperature transition from 487 to $515^{\circ} \mathrm{C}$ occurred whilst growing the material, i.e. without any growth interruption. To further improve the material quality, five sets of strained $10 \mathrm{~nm}$ AlSb/ $10 \mathrm{~nm} \mathrm{GaSb}$ dislocation filter superlattices (DFSLs) were grown on top of the two-step GaSb buffer layer. The final threading dislocation density was found to be $\sim 3 \times 10^{7} \mathrm{~cm}^{-2}$ with no APDs.

Photoluminescene (PL) measurements of the as-grown wafer were carried out in a continuous flow He cryostat, using a $785 \mathrm{~nm}$ diode laser, a Bruker Vertex 70 Fourier Transform Infrared (FTIR) Spectrometer, $77 \mathrm{~K}$ InSb photodiode detector and lock-in amplifier. The structure was then processed into mesa-etched photodetectors using conventional photolithography and processing techniques. Photoresponse measurements were performed using an OptistatDN-V2 cryostat and an FTIR spectrometer. Electrical measurements were made using a Lakeshore TTPX low-temperature probe station, equipped with a radiation shield and a Keithley source measurement unit.

\section{DESIGN AND FABRICATION OF SUPERLATTICE NBN DETECTOR ON SILICON}

Following the growth of the $\mathrm{GaSb} / \mathrm{Si}$ buffer, the substrate temperature was lowered to $430{ }^{\circ} \mathrm{C}$ for the growth of the superlattice $\mathrm{nBn}$ detector (figure 1(a)). The absorber layer superlattice (ALSL) and contact layer superlattice (CLSL) consist of 320 and 30 repeats of the InAs/InAsSb layers respectively, with a period thickness of $13 \mathrm{~nm}$ (InAs=5.8 nm, InAsSb=7.2 nm) and $\mathrm{Sb}$ content of $16.5 \%$. A growth rate of $1 \mathrm{ML} / \mathrm{s}$ was used both for the InAs and InAsSb layer. The contact and absorber layers were intentionally doped using Te: $2 \times 10^{17} \mathrm{~cm}^{-3}$ and $5 \times 10^{16} \mathrm{~cm}^{-3}$ respectively. An optimized shutter sequence was used to create abrupt InAs/InAsSb interfaces. This sequence consisted of exposing the InAs surface to an As flux for 10 seconds, followed by 3 seconds of simultaneous exposure to As and Sb, as shown in Figure 1(b). A growth rate of approximately $1 \mathrm{ML} / \mathrm{s}$ was used for the growth of the $100 \mathrm{~nm}$ thick $\mathrm{Al}_{0.9} \mathrm{Ga}_{0.1} \mathrm{As}_{0.1} \mathrm{Sb}_{0.9}$ electron blocking barrier. The AlGaAsSb layer provides a high conduction band offset (Figure 1c) which blocks the flow of the dark current carriers (electrons), while enabling the unimpeded flow of the photogenerated holes towards the contact layer.

A high quality InAs/InAsSb SL requires strain balancing using an optimized combination of layer thicknesses and $\mathrm{Sb}$ composition in the InAsSb layer. The bandgap of the SL and hole localization energy mainly depends on the Sb composition and the thickness of the InAsSb layer, which introduces compressive strain ${ }^{7}$. This must be compensated by the tensile strain in the InAs layer, which determines the electron energy. All parameters need to be simultaneously optimized to control strain balancing, spectral response and absorption strength (which is proportional to the electron/hole wavefunction overlap). The thickness of the individual layers must also be smaller than the critical layer thickness to avoid generating additional defects. The quantum confinement levels of the InAs/InAsSb QWs structure were calculated using Nextnano software, assuming symmetrically rectangular QWs. Material parameters for bulk InAs and InSb were taken from the work reported by Vurgaftman et $\mathrm{al}^{8}$. The lattice constant of InAsSb alloys were obtained using Vegard's law:

$$
\mathrm{a}\left(\operatorname{InAs}_{1-\mathrm{x}} \mathrm{Sb}_{\mathrm{x}}\right)=\mathrm{xa}(\operatorname{InSb})+(1-\mathrm{x}) \mathrm{a}(\operatorname{In} A s)
$$




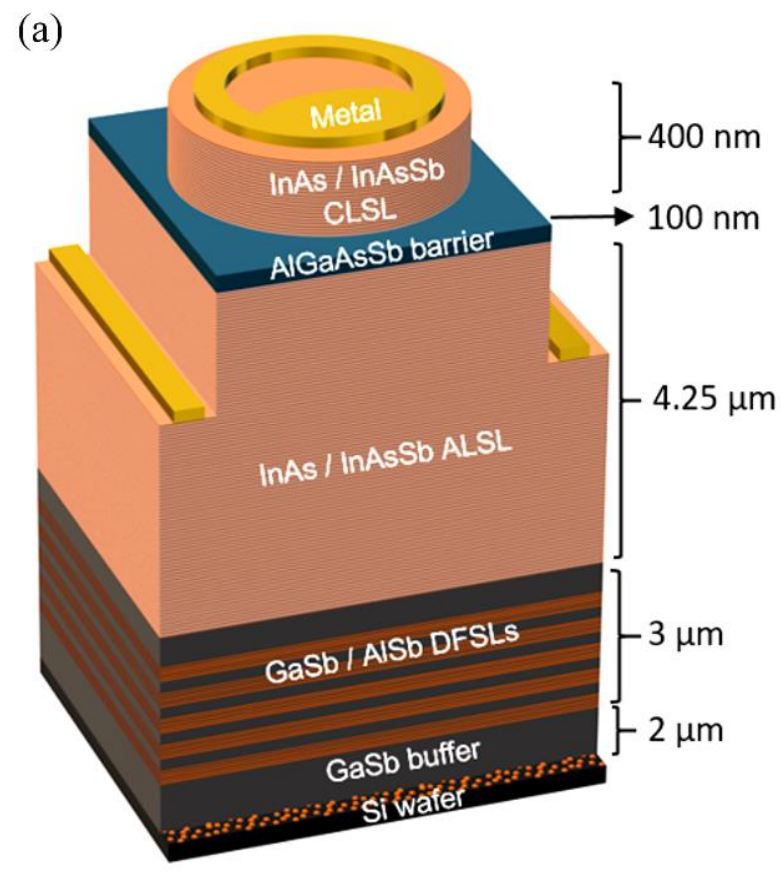

\begin{tabular}{|c|c|}
\hline $5.8 \mathrm{~nm}$ InAs barrier & In On, As On, Sb Off \\
\hline $10 \mathrm{sec}$ As soaking & In Off, As On, Sb Off \\
\hline $7.2 \mathrm{~nm}$ InAsSb well & In On, As On, Sb On \\
\hline $3 \mathrm{sec}$ As and Sb soaking & In Off, As On, Sb On \\
\hline $10 \mathrm{sec}$ As soaking & In Off, As On, Sb Off \\
\hline $5.8 \mathrm{~nm}$ InAs barrier & In On, As On, Sb Off \\
\hline
\end{tabular}

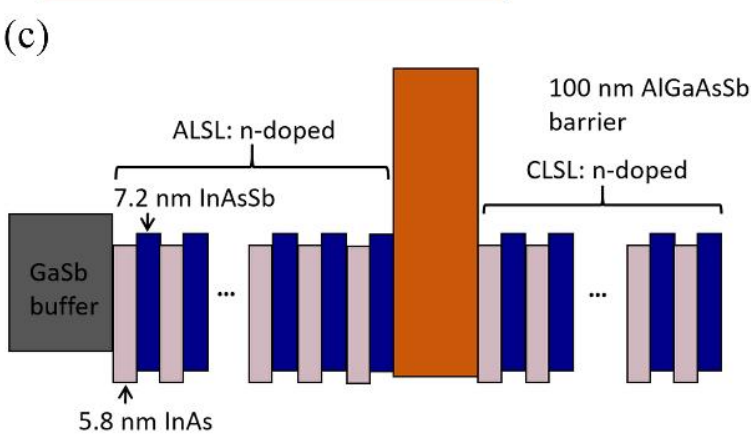

Figure 1. (a) Schematic illustration of the InAs/InAsSb SL nBn detector grown on top of the GaSb/Si buffer. (b) Diagram of the shutter sequence used for the growth of the InAs/InAsSb superlattice. (c) A schematic diagram of the InAsSb/InAs SL $\mathrm{nBn}$ detector showing the high conduction band offset provided by the AlGaAsSb barrier layer.

(a)

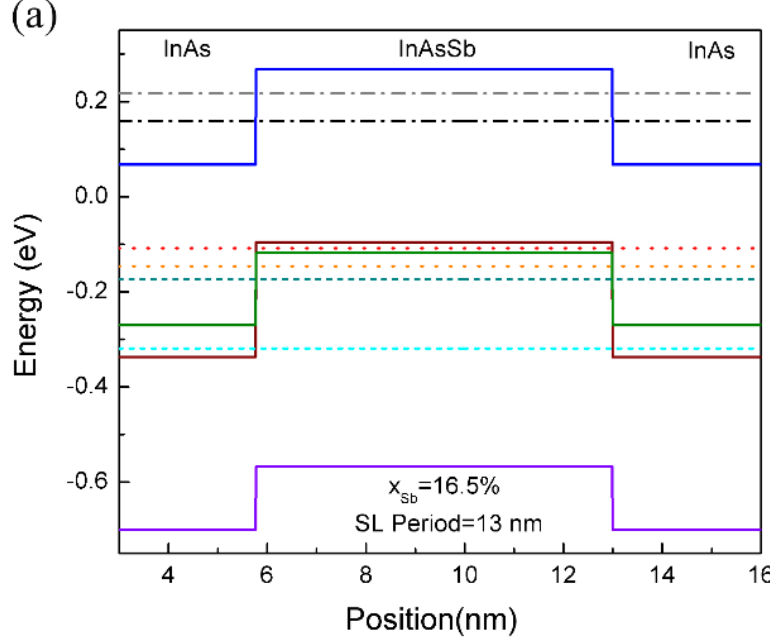

(b)

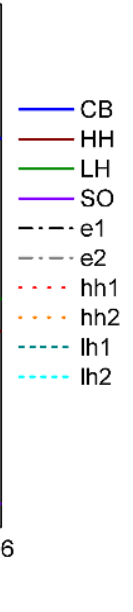

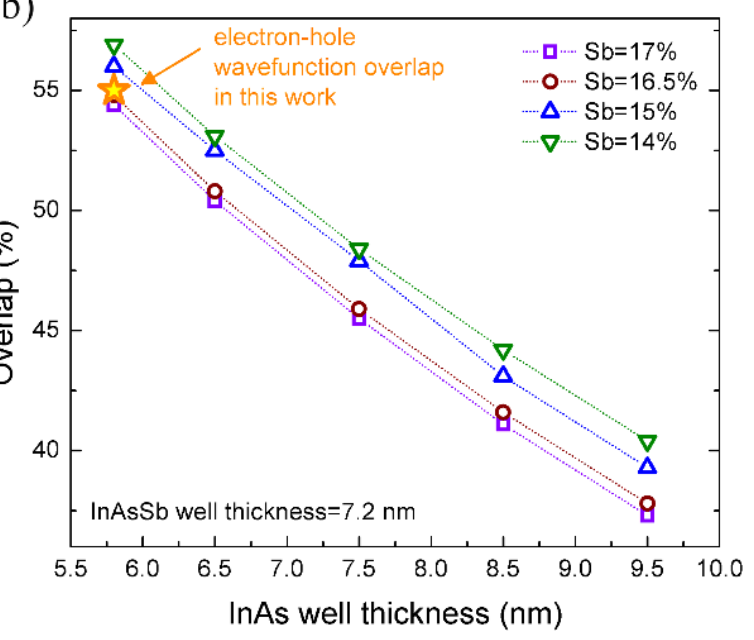

Figure 2. (a) Calculated InAs/InAsSb SL band structure showing the electron and hole levels at low temperature (5 K). (b) Calculated wavefunction overlap of the ground state transition as a function of the Sb content and thickness of the InAs well. 
by interpolation of values between InAs and InSb binary alloys. The band gap energy $\mathrm{E}_{\mathrm{g}}$ was obtained using the quadratic approximation:

$$
\mathrm{E}_{\mathrm{g}}\left(\operatorname{InAs}_{1-\mathrm{x}} \mathrm{Sb}_{\mathrm{x}}\right)=\mathrm{x} \mathrm{E}_{\mathrm{g}}(\operatorname{InSb})+(1-\mathrm{x}) \mathrm{E}_{\mathrm{g}}(\operatorname{InAs})+\mathrm{x}(1-\mathrm{x}) \mathrm{C},
$$

where $\mathrm{C}$ is the bowing parameter for InAsSb. A value of $0.971 \mathrm{eV}$ was used for the total bowing parameter, as reported by Webster et. al. ${ }^{9}$. Figure 2(a) shows the calculated bandstructure at $5 \mathrm{~K}$. The electron-hole wavefunction overlap, $F$, shown in figure 2(b), was calculated using:

$$
\mathrm{F}=\int \Psi_{\mathrm{h}}^{*}(\mathrm{x}, \mathrm{y}, \mathrm{z}) \cdot \Psi_{\mathrm{e}}(\mathrm{x}, \mathrm{y}, \mathrm{z}) \mathrm{d}^{3}(\mathrm{x}, \mathrm{y},)
$$

where $\Psi_{\mathrm{h}}$ and $\Psi_{\mathrm{e}}$ are the hole and electron wavefunctions respectively. Figure $2 \mathrm{~b}$ shows that the use of thicker InAs QWs decreases the wavefunction overlap. However, the thickness of the InAsSb QW should also be carefully chosen as very thin layers $(<5 \mathrm{~nm})$ can suffer from inhomogeneous distribution of Sb. For the SL structure used in this work, the groundstate transition (e1 to hh1 band) energy was calculated as $268 \mathrm{meV}(4.63 \mu \mathrm{m})$ with an electron-hole wavefunction overlap of $55 \%$.

\section{PHOTOLUMINESCENCE}

Power dependent PL measurements were obtained for an unprocessed specimen at low temperature (5 K), as shown in Figure 3(a). The signal peaks at $4.7 \mu \mathrm{m}$ (for a laser power of $2.5 \mathrm{~W} / \mathrm{cm}^{2}$ ) which corresponds to a transition energy of 264 $\mathrm{meV}$ (Peak I). This value is in excellent agreement to the theoretical ground-state transition energy calculated earlier (268 $\mathrm{meV}$ ). The spectra also exhibited a second peak, noted as Peak II, centred at around $243 \mathrm{meV}$. This value is $21 \mathrm{meV}$ lower compared to the ground-state transition energy, suggesting a middle-gap donor or acceptor level related recombination. For wavelengths longer than $5.3 \mu \mathrm{m}$ the signal is affected by the cut-off wavelength limit of the cryostat window. The intensity of Peak I decreased by approximately 40 times and demonstrated a small red shift of $1.5 \mathrm{meV}$ as the laser excitation power was reduced down to $0.1 \mathrm{~W} / \mathrm{cm}^{2}$. This is attributed to the spatial separation of charge carriers due to the type-II band alignment and the formation of a triangular potential well at the superlattice interfaces. Similar PL behaviour has been reported previously for MIR InAs/InAsSb superlattices grown on GaSb substrates ${ }^{10}$. Furthermore, at low laser excitation powers the intensity of Peak II becomes significantly lower confirming the origin of the peak being from donoracceptor related recombination. Figure 3(b) shows the dependence of the integrated PL intensity of Peak I to the laser excitation power at $5 \mathrm{~K}$. This data was fitted using the equation:

$$
\mathrm{I}_{\mathrm{PL}}=\mathrm{CP}_{\text {laser }}^{\gamma}
$$

where $\mathrm{P}_{\text {laser }}$ is the laser excitation power, $\gamma$ is the gradient of the slope and $\mathrm{c}$ is a constant ${ }^{11}$. Parameter $\gamma$ is related to the dominant recombination process. A $\gamma$ value close to $1 / 2,1$ or $3 / 2$ indicates that the dominant recombination process is $\mathrm{SRH}$, radiative or Auger recombination respectively. The gradient, $\gamma$, of the slope is calculated to be 1.08 indicating that radiative recombination is the dominant recombination mechanism, despite the presence of dislocations inside the absorption layer. This could be attributed to the position of the localized defect states above the conduction band for a Gafree type-II superlattices suggesting a defect-tolerant structure. Temperature dependent PL was also measured for the sample, as shown in Figure 3(c). With increasing temperature, up to $300 \mathrm{~K}$, the main peak demonstrated a red shift and eventually moved beyond the cut-off wavelength limit $(5.3 \mu \mathrm{m})$ of the cryostat window for temperatures higher than 200 K. Furthermore, the intensity of the low energy peak was significantly decreased with increasing temperature, and for temperatures higher than $40 \mathrm{~K}$ the peak could no longer be identified in the spectra, consistent with donor-acceptor recombination in InAs-based materials. 

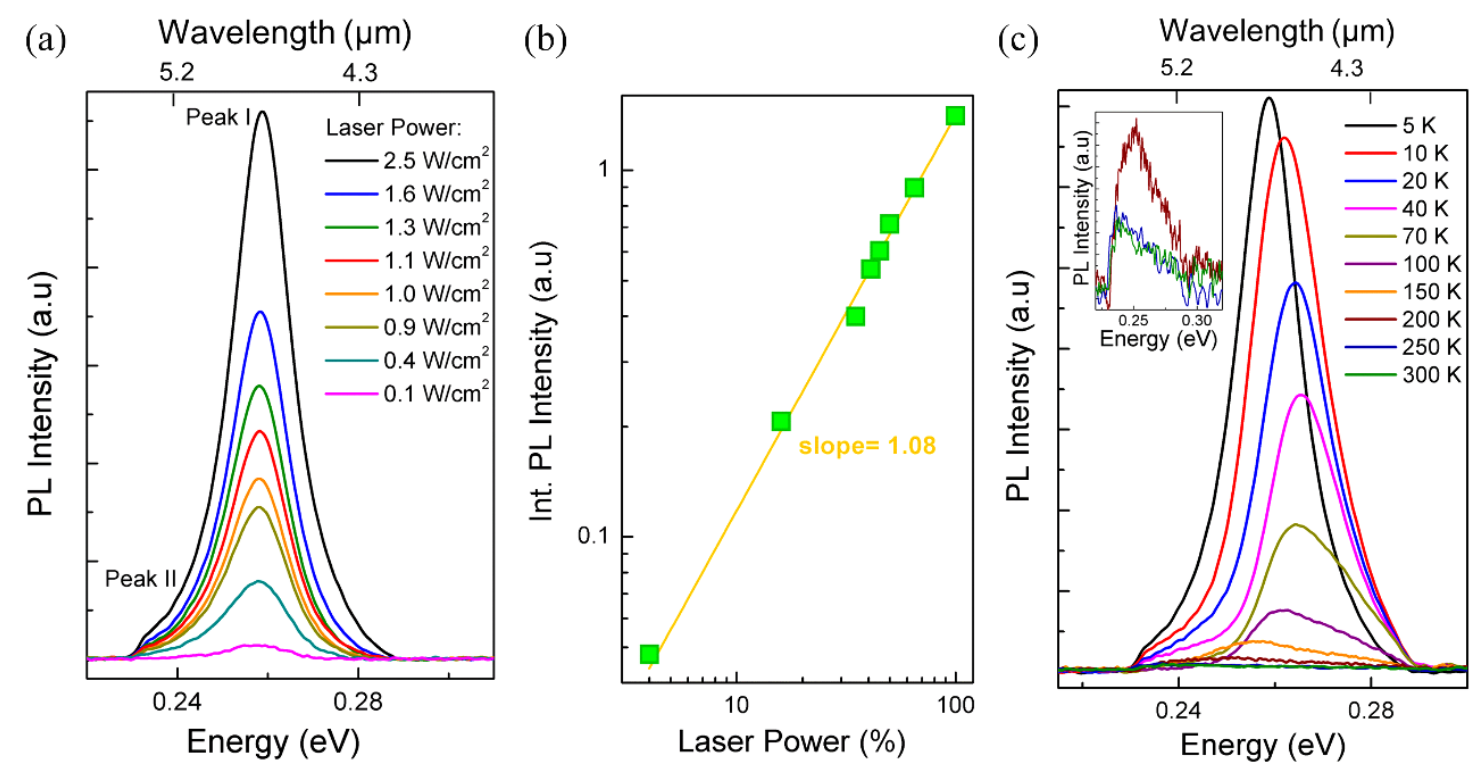

Figure 3. (a) Power depended PL spectra measurements obtained at 5 K. (b) Integrated PL intensity dependence on the laser excitation power. (c) Temperature dependent PL spectra measured for the sample. The inset figure highlights that the high temperature spectra ( 200 to $300 \mathrm{~K}$ ) have moved beyond the cut-off wavelength of the cryostat window.

\section{ELECTRICAL AND OPTICAL CHARACTERISATION}

Electrical characterisation of detector devices with a mesa diameter of $50 \mu \mathrm{m}$ was performed in the temperature range from 110 to $300 \mathrm{~K}$, as shown in Figure 4(a). The dark current density increased by approximately 5 orders of magnitude with increasing temperature, from $1.14 \times 10^{-5} \mathrm{Acm}^{-2}$ at $110 \mathrm{~K}$ to $8.65 \times 10^{-1} \mathrm{Acm}^{-2}$ at room temperature. The dark current density data for a reverse bias of $100 \mathrm{mV}$ presented in Figure 4(b) was fitted using the equation ${ }^{12}$ :

$$
\mathrm{J}_{\text {dark }}=\mathrm{T}^{3 / 2} \exp \left(-\mathrm{E}_{\mathrm{a}} / \mathrm{kT}\right)
$$

to determine the dominant dark current generation mechanism where $E_{a}$ is the activation energy of the dark current mechanism. Depending on whether the activation energy is close to $\mathrm{E}_{\mathrm{g}, 0 \mathrm{~K}}$ or $\mathrm{E}_{\mathrm{g}} / 2$ the dominant dark current mechanism is diffusion or generation-recombination respectively. The fit indicated an activation energy of $173 \mathrm{meV}$. This value is 39 $\mathrm{meV}$ higher than half the ground state transition calculated earlier $(268 \mathrm{meV})$, suggesting that SRH generationrecombination dark currents dominate for temperatures higher than $110 \mathrm{~K}$, with a small additional contribution from diffusion.

Figure 5(a) shows the normalized spectral response of the detector obtained for the temperature range between 77 to $300 \mathrm{~K}$ under a bias operation of $\mathrm{V}_{\mathrm{b}}=-100 \mathrm{mV}$. The device demonstrated a strong photoresponse signal covering the spectral range from 2.5 to $6 \mu \mathrm{m}$. A sharp absorption dip can be clearly observed around $4.2 \mu \mathrm{m}$ corresponding to the $\mathrm{CO}_{2}$ atmospheric absorption in the optical path of the incident light beam. The 50\% cut-off wavelength varied weakly with temperature, shifting from $4.82 \mu \mathrm{m}$ at $77 \mathrm{~K}$ to $5.86 \mu \mathrm{m}$ at room temperature. The responsivity of a top-side-illuminated detector device was also measured with varying temperature for the spectral range from 3.7 to $4.9 \mu \mathrm{m}$. Isolation of the spectral range in the MIR atmospheric absorption window (3.7-4.9 $\mu \mathrm{m})$ was employed to simulate the operating conditions of typical commercial detectors. The results are shown in Figure 5(b). The detector responsivity gradually increased with temperature up to $200 \mathrm{~K}$, reaching a maximum value of $0.88 \mathrm{~A} / \mathrm{W}$. For temperatures higher than $200 \mathrm{~K}$ the responsivity significantly decreased to $0.5 \mathrm{~A} / \mathrm{W}$ at $220 \mathrm{~K}$. The maximum responsivity corresponded to a maximum external efficiency of $25.6 \%$ at $200 \mathrm{~K}$. 
(a)

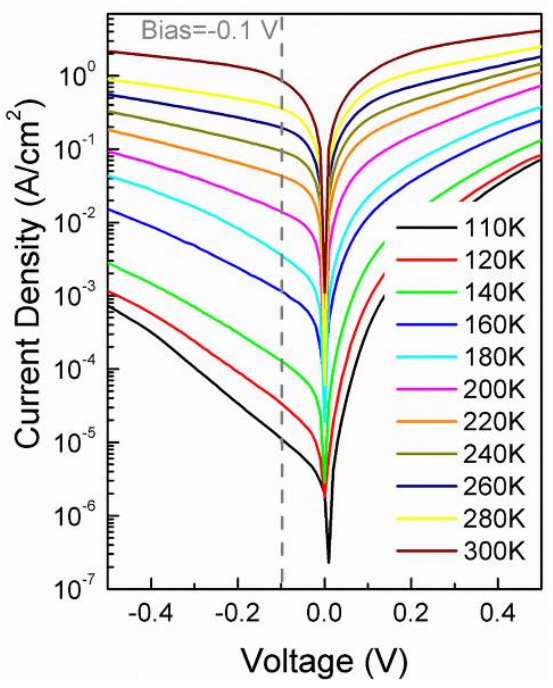

(b) Temperature $(\mathrm{K})$

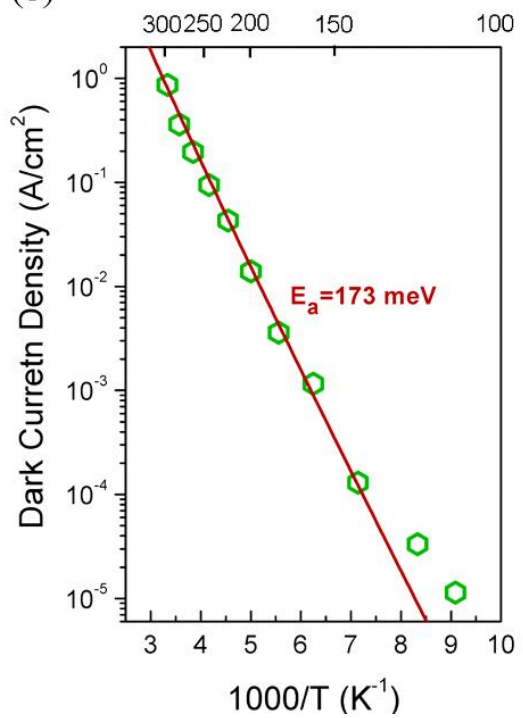

Figure 4. (a) Current-Voltage characteristics for the temperature range from 110 to $300 \mathrm{~K}$. The dashed line shows the position of the $-100 \mathrm{mV}$ reverse bias. (b) Dark current $\left(\mathrm{J}_{\text {dark }}\right)$ vs temperature obtained for a reverse bias voltage of $\mathrm{V}_{\mathrm{b}}=100 \mathrm{mV}$.

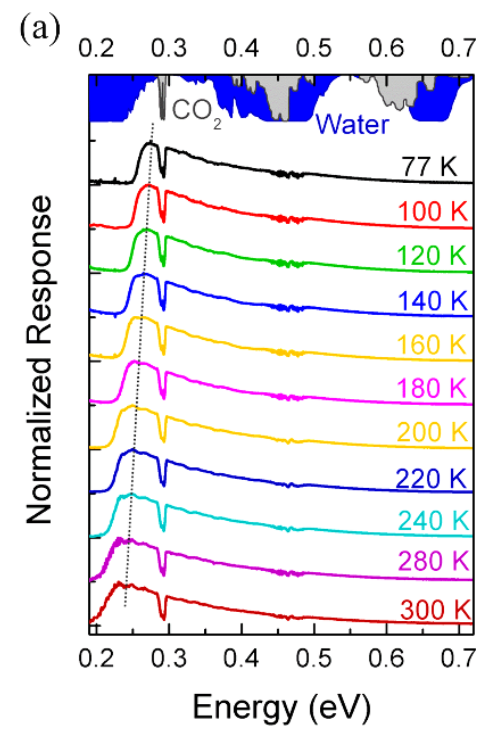

(b)

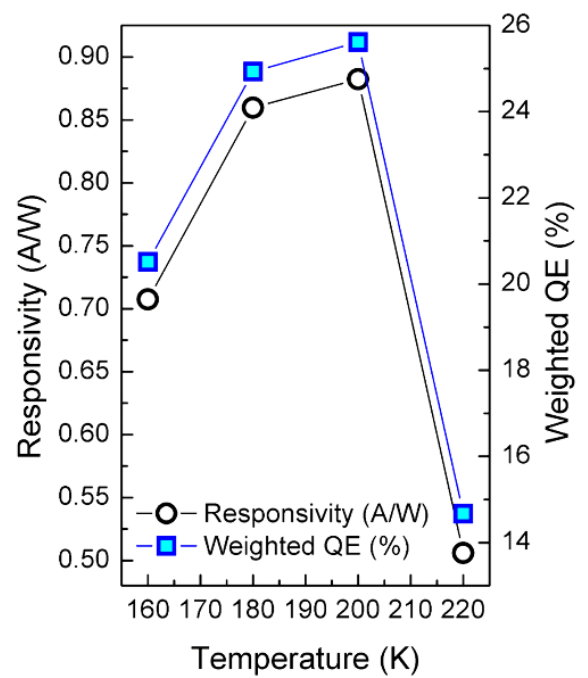

Figure 5. (a) Normalized spectral response signal at different temperatures. The $\mathrm{CO}_{2}$ (gray) and water-vapor (blue) atmospheric absorption spectra are also shown. The dotted line is a guide to the eye of the peak position. (b) Spectral responsivity and external quantum efficiency as a function of temperature. The device operated at a reverse bias of $100 \mathrm{mV}$ during the measurements.

Finally, the shot and thermal noise limited specific detectivity $\mathrm{D}^{*}$ was calculated using the dark current and responsivity data along with equation ${ }^{13}$ :

$$
\mathrm{D}^{*}=\frac{\mathrm{R}}{\sqrt{2 \mathrm{qJ} \mathrm{dark}_{\mathrm{dar}}+\frac{4 \mathrm{kT}}{\mathrm{R}_{\mathrm{d}} \mathrm{A}}}}
$$


where $\mathrm{R}$ is the responsivity in $\mathrm{A} / \mathrm{W}, \mathrm{q}$ is the elementary charge, $\mathrm{R}_{\mathrm{d}}$ the dynamic resistance and $\mathrm{A}$ the detector contact area. The temperature dependent specific detectivity data shown in Figure 6 demonstrate a maximum value of $3.65 \times 10^{10}$ Jones at $160 \mathrm{~K}$. The detectivity dropped to $1.5 \times 10^{10}$ Jones as the temperature was increased $200 \mathrm{~K}$.

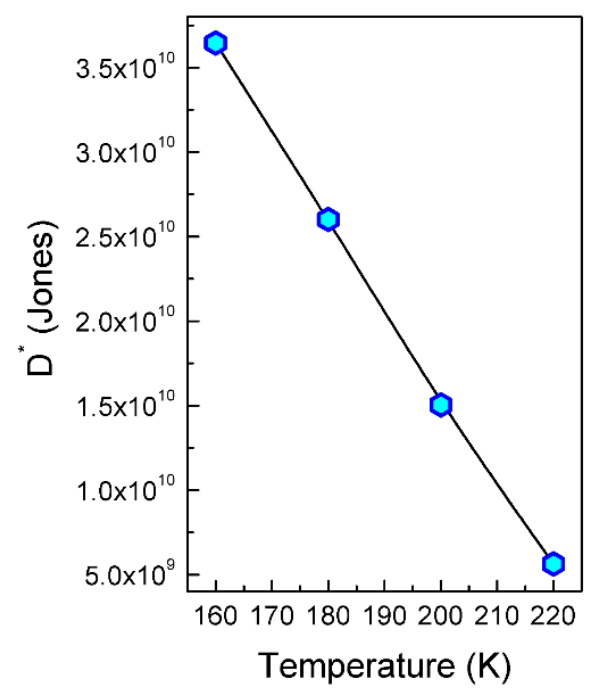

Figure 6. Temperature dependence of the SL nBn detector's specific detectivity.

\section{CONCLUSION}

A high-performance MIR type-II InAs/InAsSb SL photodetector grown on Si by MBE using a GaSb-based DFSL structure has been presented. Low-temperature photoluminescence measurements revealed that the dominant recombination mechanism was radiative and that the peak emission energy was $264 \mathrm{meV}$. Temperature dependent dark current-voltage characteristics indicated Shockley-Read-Hall as the dominant dark current mechanism when the device was operating as a detector. Nevertheless, the detector exhibited a maximum responsivity of $0.88 \mathrm{~A} / \mathrm{W}$ at $200 \mathrm{~K}$, corresponding in an external quantum efficiency of $25.6 \%$. The specific detectivity was calculated to be as high as $3.65 \times 10^{10}$ Jones at $160 \mathrm{~K}$.

\section{ACKNOWLEDGEMENTS}

Financial support for this work was provided by EPSRC [grant number EP/N018605/1]; the Royal Academy of Engineering [grant number 10216/114], the Joy Welch Educational Charitable Trust and the Lancaster University Research Committee.

\section{REFERENCES}

[1] D. Lackner, M. Steger, M. L. W. Thewalt, O. J. Pitts, Y. T. Cherng, S. P. Watkins, E. Plis, and S. Krishna, "InAs/InAsSb strain balanced superlattices for optical detectors: Material properties and energy band simulations," Journal of Applied Physics, vol. 111, no. 3, pp. 034507, 2012.

[2] Y. Aytac, B. V. Olson, J. K. Kim, E. A. Shaner, S. D. Hawkins, J. F. Klem, J. Olesberg, M. E. Flatté, and T. F. Boggess, "Bandgap and temperature dependence of Auger recombination in InAs/InAsSb type-II superlattices," Journal of Applied Physics, vol. 119, no. 21, pp. 215705, 2016. 
[3] A. D. Prins, M. K. Lewis, Z. L. Bushell, S. J. Sweeney, S. Liu, and Y.-H. Zhang, "Evidence for a defect level above the conduction band edge of InAs/InAsSb type-II superlattices for applications in efficient infrared photodetectors," Applied Physics Letters, vol. 106, no. 17, pp. 171111, 2015.

[4] B. V. Olson, E. A. Shaner, J. K. Kim, J. F. Klem, S. D. Hawkins, L. M. Murray, J. P. Prineas, M. E. Flatté, and T. F. Boggess, "Time-resolved optical measurements of minority carrier recombination in a mid-wave infrared InAsSb alloy and InAs/InAsSb superlattice,” Applied Physics Letters, vol. 101, no. 9, pp. 092109, 2012.

[5] E. Delli, V. Letka, P. D. Hodgson, E. Repiso, J. P. Hayton, A. P. Craig, Q. Lu, R. Beanland, A. Krier, A. R. J. Marshall, and P. J. Carrington, "Mid-Infrared InAs/InAsSb Superlattice nBn Photodetector Monolithically Integrated onto Silicon," ACS Photonics, vol. 6, no. 2, pp. 538-544, 2019/02/20, 2019.

[6] J. B. Rodriguez, L. Cerutti, G. Patriarche, L. Largeau, K. Madiomanana, and E. Tournié, "Characterization of antimonide based material grown by molecular epitaxy on vicinal silicon substrates via a low temperature AlSb nucleation layer," Journal of Crystal Growth, vol. 477, pp. 65-71, 2017/11/01/, 2017.

[7] A. Haddadi, G. Chen, R. Chevallier, A. M. Hoang, and M. Razeghi, "InAs/InAs1-xSbx type-II superlattices for high performance long wavelength infrared detection," Applied Physics Letters, vol. 105, no. 12, pp. 121104, 2014.

[8] I. Vurgaftman, J. R. Meyer, and L. R. Ram-Mohan, "Band parameters for III-V compound semiconductors and their alloys," Journal of Applied Physics, vol. 89, no. 11, pp. 5815-5875, 2001.

[9] P. T. Webster, N. A. Riordan, S. Liu, E. H. Steenbergen, R. A. Synowicki, Y.-H. Zhang, and S. R. Johnson, "Absorption properties of type-II InAs/InAsSb superlattices measured by spectroscopic ellipsometry," Applied Physics Letters, vol. 106, no. 6, pp. 061907, 2015.

[10] E. Steenbergen, J. Massengale, V. Cowan, Z. Lin, Y.-H. Zhang, and C. Morath, Proton radiation effects on the photoluminescence of infrared InAs/InAsSb superlattices, $\mathrm{p} . \wedge$ pp. OPO: SPIE, 2013.

[11] J.-B. Wang, D. Ding, S. R. Johnson, S.-Q. Yu, and Y.-H. Zhang, "Determination and improvement of spontaneous emission quantum efficiency in GaAs/AlGaAs heterostructures grown by molecular beam epitaxy," physica status solidi (b), vol. 244, no. 8, pp. 2740-2751, 2007.

[12] P. Klipstein, O. Klin, S. Grossman, N. Snapi, B. Yaakobovitz, M. Brumer, I. Lukomsky, D. Aronov, M. Yassen, B. Yofis, A. Glozman, T. Fishman, E. Berkowitz, O. Magen, I. Shtrichman, and E. Weiss, MWIR InAsSb XBn detectors for high operating temperatures, p.^pp. SI: SPIE, 2010.

[13] A. P. Craig, A. R. J. Marshall, Z.-B. Tian, S. Krishna, and A. Krier, "Mid-infrared InAs0.79Sb0.21-based nBn photodetectors with $\mathrm{Al} 0.9 \mathrm{Ga} 0.2 \mathrm{As} 0.1 \mathrm{Sb} 0.9$ barrier layers, and comparisons with InAs0.87Sb0.13 p-i-n diodes, both grown on GaAs using interfacial misfit arrays," Applied Physics Letters, vol. 103, no. 25, pp. 253502, 2013. 